\title{
Front Matter: Volume 9080
}

, "Front Matter: Volume 9080," Proc. SPIE 9080, Laser Radar Technology and Applications XIX; and Atmospheric Propagation XI, 908001 (26 June 2014); doi: $10.1117 / 12.2074224$

SPIE. Event: SPIE Defense + Security, 2014, Baltimore, MD, United States 


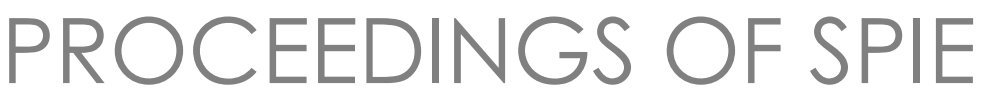

\section{Laser Radar Technology and Applications XIX; and Atmospheric Propagation XI}

Monte D. Turner

Gary W. Kamerman

Linda M. Wasiczko Thomas

Earl J. Spillar

Editors

6-7 May 2014

Baltimore, Maryland, United States

Sponsored and Published by

SPIE 
The papers included in this volume were part of the technical conference cited on the cover and title page. Papers were selected and subject to review by the editors and conference program committee. Some conference presentations may not be available for publication. The papers published in these proceedings reflect the work and thoughts of the authors and are published herein as submitted. The publisher is not responsible for the validity of the information or for any outcomes resulting from reliance thereon.

Please use the following format to cite material from this book:

Author(s), "Title of Paper," in Laser Radar Technology and Applications XIX; and Atmospheric Propagation XI, edited by Monte D. Turner, Gary W. Kamerman, Linda M. Wasiczko Thomas, Earl J. Spillar, Proceedings of SPIE Vol. 9080 (SPIE, Bellingham, WA, 2014) Article CID Number.

ISSN: 0277-786X

ISBN: 9781628410174

Published by

SPIE

P.O. Box 10, Bellingham, Washington 98227-0010 USA

Telephone +1 3606763290 (Pacific Time) · Fax +1 3606471445

SPIE.org

Copyright (C) 2014, Society of Photo-Optical Instrumentation Engineers.

Copying of material in this book for internal or personal use, or for the internal or personal use of specific clients, beyond the fair use provisions granted by the U.S. Copyright Law is authorized by SPIE subject to payment of copying fees. The Transactional Reporting Service base fee for this volume is $\$ 18.00$ per article (or portion thereof), which should be paid directly to the Copyright Clearance Center (CCC), 222 Rosewood Drive, Danvers, MA 01923. Payment may also be made electronically through CCC Online at copyright.com. Other copying for republication, resale, advertising or promotion, or any form of systematic or multiple reproduction of any material in this book is prohibited except with permission in writing from the publisher. The CCC fee code is 0277-786X/14/\$18.00.

Printed in the United States of America.

Publication of record for individual papers is online in the SPIE Digital Library.

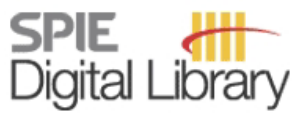

SPIEDigitalLibrary.org

Paper Numbering: Proceedings of SPIE follow an e-First publication model, with papers published first online and then in print and on CD-ROM. Papers are published as they are submitted and meet publication criteria. A unique, consistent, permanent citation identifier (CID) number is assigned to each article at the time of the first publication. Utilization of CIDs allows articles to be fully citable as soon as they are published online, and connects the same identifier to all online, print, and electronic versions of the publication. SPIE uses a six-digit CID article numbering system in which:

- The first four digits correspond to the SPIE volume number.

- The last two digits indicate publication order within the volume using a Base 36 numbering

system employing both numerals and letters. These two-number sets start with 00, 01, 02, 03, 04, $05,06,07,08,09,0 A, 0 B \ldots$. 0Z, followed by 10-1Z, 20-2Z, etc.

The CID Number appears on each page of the manuscript. The complete citation is used on the first page, and an abbreviated version on subsequent pages. Numbers in the index correspond to the last two digits of the six-digit CID Number. 


\section{Contents}

ix Conference Committee

\section{Part A Laser Radar Technology and Applications XIX}

\section{SESSION $1 \quad$ ADVANCED SYSTEMS AND COMPONENTS I}

908002 Multi-dimensional laser radars (Invited Paper) [9080-1]

V. Molebny, Academy of Technological Sciences of Ukraine (Ukraine); O. Steinvall, Swedish Defence Research Agency (Sweden)

908004 A polarimetric scanning LADAR: system development and performance analysis [9080-3] Y. Y. Markushin, N. P. Calvano, G. S. Pati, R. Tripathi, Delaware State Univ. (United States)

908005 A long-distance laser altimeter for terrain relative navigation and spacecraft landing [9080-4]

D. F. Pierrottet, Coherent Applications, Inc. (United States); F. Amzajerdian, B. Barnes, NASA Langley Research Ctr. (United States)

$908006 \quad 1541 \mathrm{~nm}$ GmAPD LADAR system [9080-5]

M. R. Kutteruf, P. Lebow, U.S. Naval Research Lab. (United States)

908007 Mosaic active imaging: direct physical modelling and image reconstruction [9080-6] E. Thouin, ONERA (France) and Institut Supérieur l'Aéronautique et de l'Espace, CNRS, Univ. de Toulouse (France); M.-T. Velluet, D. Hamoir, L. Hespel, ONERA (France); F. Malgouyres, Institut de Mathématiques de Toulouse, CNRS, Univ. de Toulouse (France); X. Briottet, ONERA (France)

\section{SESSION 2 ADVANCED SYSTEMS AND COMPONENTS II}

$908008 \quad 16 \mathrm{~W}$ average power $2 \mu \mathrm{m}$ Thulium fiber laser with one stage MOPA [9080-9]

W. Wu, Shanghai Institute of Optics and Fine Mechanics (China) and Qufu Normal Univ. (China); T. YU, Q. Huang, X. Cheng, W. Chen, Shanghai Institute of Optics and Fine Mechanics (China)

9080 OA Development of scanning laser sensor for underwater 3D imaging with the coaxial optics [9080-11]

H. Ochimizu, M. Imaki, S. Kameyama, Mitsubishi Electric Corp. (Japan); T. Saito, Mitsubishi Electric Tokki Systems Corp. (Japan); S. Ishibashi, H. Yoshida, Japan Agency for MarineEarth Science and Technology (Japan) 
$90800 \mathrm{C}$ Outward atmospheric scintillation effects and inward atmospheric scintillation effects comparisons for direct detection ladar applications [9080-13]

D. G. Youmans, Parsons Corp. (United States)

9080 OD High-fidelity flash lidar model development [9080-14]

G. D. Hines, NASA Langley Research Ctr. (United States); D. F. Pierrottet, Coherent Applications, Inc. (United States); F. Amzajerdian, NASA Langley Research Ctr. (United States)

9080 OE Doppler lidar system design via interdisciplinary design concept at NASA Langley Research Center: Part I [9080-16]

C. M. Boyer, NASA Langley Research Ctr. (United States); T. P. Jackson, Old Dominion Univ. (United States); J. Y. Beyon, L. B. Petway, NASA Langley Research Ctr. (United States)

9080 OF Design and performance of a fiber array coupled multi-channel photon counting, 3D imaging, airborne lidar system [9080-8]

G. Huang, R. Shu, L. Hou, M. Li, Shanghai Institute of Technical Physics (China)

9080 0G Doppler lidar system design via interdisciplinary design concept at NASA Langley Research Center: Part II [9080-900]

A. I. Crasner, Univ. of Michigan (United States); S. Scola, J. Y. Beyon, L. B. Petway, NASA Langley Research Ctr. (United States)

$9080 \mathrm{OH}$ Doppler lidar system design via interdisciplinary design concept at NASA Langley Research Center: Part III [9080-901]

B. W. Barnes, NASA Langley Research Ctr. (United States); A. M. Sessions, The Univ. of Arizona (United States); J. Y. Beyon, L. B. Petway, NASA Langley Research Ctr.

(United States)

\section{SESSION 3 SIGNAL PROCESSING}

9080 ol Improving waveform lidar processing toward robust deconvolution of signals for improved structural assessments [9080-17]

K. Cawse-Nicholson, J. van Aardt, S. Hagstrom, P. Romanczyk, Rochester Institute of Technology (United States); C. Schaaf, Univ. of Massachusetts Boston (United States); A. Strahler, Z. Li, Boston Univ. (United States); K. Krause, National Ecological Observatory Network (United States)

$9080 \mathrm{0J}$ Online waveform processing for demanding target situations [9080-18]

M. Pfennigbaver, C. Wolf, J. Weinkopf, A. Ullrich, RIEGL Laser Measurement Systems GmbH (Austria)

9080 OK Range resolution improvement of eyesafe ladar testbed (ELT) measurements using sparse signal deconvolution [9080-19]

S. E. Budge, J. H. Gunther, Utah State Univ. (United States)

$9080 \mathrm{OL} \quad H i g h-s p e e d$ on-board data processing for science instruments [9080-21]

J. Y. Beyon, T.-K. Ng, B. Lin, Y. Hu, W. Harrison, NASA Langley Research Ctr. (United States) 
$90800 \mathrm{M}$ Incoherent pulse compression in laser range finder [9080-22]

D. Grodensky, D. Kravitz, N. Arbel, Bar-Ilan Univ. (Israel); N. Levanon, Tel Aviv Univ. (Israel);

A. Zadok, Bar-llan Univ. (Israel)

\section{SESSION 4 DATA PROCESSING AND ANALYSIS I}

9080 ON Algorithm for detecting important changes in lidar point clouds [9080-23]

D. Korchev, Y. Owechko, HRL Labs., LLC (United States)

908000 Improved registration for 3D image creation using multiple texel images and incorporating low-cost GPS/INS measurements [9080-24]

S. E. Budge, X. Xie, Utah State Univ. (United States)

9080 OP Estimating sampling completeness of lidar datasets using voxel-based geometry [9080-25]

S. Hagstrom, D. Messinger, K. Salvaggio, Rochester Institute of Technology

(United States)

90800 Graph segmentation and support vector machines for bare earth classification from lidar [9080-26]

N. S. Shorter, O. Smith, P. Smith, M. Rahmes, Harris Corp. (United States)

9080 OR Comparison of lidar and stereo photogrammetric point clouds for change detection [9080-27]

P. L. Basgall, National Geospatial-Intelligence Agency (United States); F. A. Kruse,

R. C. Olsen, Naval Postgraduate School (United States)

\section{SESSION 5 DATA PROCESSING AND ANALYSIS II}

9080 OS Uncertainty assessment and probabilistic change detection using terrestrial and airborne lidar [9080-28]

A. Jalobeanu, Univ. of Texas at Austin (United States) and Naval Postgraduate School

(United States); A. M. Kim, S. C. Runyon, R. C. Olsen, F. A. Kruse, Naval Postgraduate School (United States)

9080 OT lidar change detection using building models [9080-29]

A. M. Kim, S. C. Runyon, Naval Postgraduate School (United States); A. Jalobeanu, Univ. of Texas at Austin (United States) and Naval Postgraduate School (United States);

C. H. Esterline, F. A. Kruse, Naval Postgraduate School (United States);

9080 OU Correlation between lidar-derived intensity and passive optical imagery [9080-30]

J. P. Metcalf, A. M. Kim, F. A. Kruse, R. C. Olsen, Naval Postgraduate School (United States)

9080 0V A calibration method of the multi-channel imaging lidar [9080-31]

W. XU, Shanghai Institute of Technical Physics (China); J. Liu, Zhengzhou Institute of Surveying and Mapping (China); R. Shu, Shanghai Institute of Technical Physics (China) 
9080 0W Using an eyesafe military laser range finder for atmospheric sensing [9080-32]

O. Steinvall, R. Persson, F. Berglund, O. Gustafsson, F. Gustafsson, Swedish Defence Research Agency (Sweden)

9080 0X Development of a fluorescence lidar for measurement of atmospheric formaldehyde [9080-33]

A. Radhakrishnan Mylapore, MassTech Inc. (United States); M. Yakshin, A. Achey, I. H. Hwang, S. Lee, N. Mehta, G. K. Schwemmer, C. R. Prasad, Science and Engineering Services, Inc. (United States); T. F. Hanisco, NASA Goddard Space Flight Ctr. (United States)

9080 OY A three-beam aerosol backscatter correlation lidar for three-component wind profiling [9080-34]

A. Radhakrishnan Mylapore, MassTech Inc. (United States); G. K. Schwemmer, C. R. Prasad, S. Lee, A. Achey, I. H. Hwang, N. Mehta, M. Yakshin, Science and Engineering Services, Inc. (United States); K. Novoselov, MassTech Inc. (United States); N. S. Prasad, NASA Langley Research Ctr. (United States)

$90800 Z \quad$ Laser remote sensing of species concentrations and dynamical processes [9080-35] C. R. Philbrick, H. D. Hallen, North Carolina State Univ. (United States)

908010 Remote aerosol species-identification using IR scattering spectroscopy [9080-36] S. Niu, C. R. Philbrick, H. D. Hallen, North Carolina State Univ. (United States)

908011 Offshore wind measurements using Doppler aerosol wind lidar (DAWN) at NASA Langley Research Center [9080-37]

J. Y. Beyon, G. J. Koch, M. J. Kavaya, NASA Langley Research Ctr. (United States)

908012 Clipping of TE- $\mathrm{CO}_{2}$ laser pulse using gas breakdown technique for high spatial resolution gas plume detection [9080-38]

T. Gasmi, Saint Louis Univ., - Madrid Campus (Spain)

\section{Part B Atmospheric Propagation XI}

\section{SESSION 7 PROPAGATION THEORY AND VALIDATION}

908013 Scintillation fluctuations of optical communication lasers in atmospheric turbulence [9080-39]

M. G. Panich, J. T. Coffaro, S. B. Belichki, L. J. Splitter, R. L. Phillips, L. C. Andrews, Kennedy Space Ctr. (United States) and CREOL, The College of Optics and Photonics, Univ. of Central Florida (United States); W. Fountain, Wayne Analytics LLC (United States);

F. M. Tucker, U.S. Army RDECOM/STTC (United States)

908014 Internal anisotropy of the turbulent scintillations [9080-40]

M. Charnotskii, Zel Technologies, LLC (United States) and National Oceanic and Atmospheric Administration (United States) 
908015 Simulation of partially spatially coherent laser beam and comparison with field test data for both terrestrial and maritime environments [9080-41]

N. Mosavi, Johns Hopkins Univ. Applied Physics Lab., LLC (United States) and Univ. of Maryland, Baltimore County (United States); C. Nelson, U.S. Naval Academy (United States); B. S. Marks, B. G. Boone, Johns Hopkins Univ. Applied Physics Lab., LLC (United States); C. R. Menyuk, Univ. of Maryland, Baltimore County (United States)

\section{SESSION 8 MITIGATION TECHNIQUES I}

908017 Atmospheric turbulence effects on a monostatic and bistatic retroreflecting link [9080-43] R. Mahon, M. S. Ferraro, P. G. Goetz, C. I. Moore, J. Murphy, W. S. Rabinovich, U.S. Naval Research Lab. (United States)

908018 Demonstrating capacity-approaching FSO communications [9080-44] M. P. Fitz, T. R. Halford, C. Kose, J. Cromwell, S. Gordon, TrellisWare Technologies, Inc. (United States)

908019 Implementation and performance of stochastic parallel gradient descent algorithm for atmospheric turbulence compensation [9080-45]

G. A. Finney, C. Persons, S. Henning, IERUS Technologies Inc. (United States); J. Hazen,

D. Whitley, The Univ. of Alabama in Huntsville (United States)

$90801 \mathrm{~A}$ Image processing techniques for laser propagation through atmospheric turbulence [9080-46]

S. B. Belichki, L. J. Splitter, L. C. Andrews, R. L. Phillips, J. T. Coffaro, M. G. Panich, CREOL, The College of Optics and Photonics, Univ. of Central Florida (United States)

\section{SESSION 9 MITIGATION TECHNIQUES II}

9080 1B Diversity effects in modulating retro-reflector links [9080-47]

W. S. Rabinovich, R. Mahon, M. S. Ferraro, P. G. Goetz, J. Murphy, U.S. Naval Research Lab. (United States)

9080 ID Integration of a concentric five element InAIAs/InGaAs avalanche photodiode array in a stabilized bi-static optical assembly [9080-49]

M. S. Ferraro, R. Mahon, W. S. Rabinovich, J. L. Murphy, U.S. Naval Research Lab. (United States); W. T. Freeman, S. Frawley, Smart Logic, Inc. (United States); P. G. Goetz, H. R. Burris, L. M. Thomas, U.S. Naval Research Lab. (United States); W. R. Clark, W. D. Waters,

K. Vaccaro, B. D. Krejca, OptoGration Inc. (United States); B. M. Mathieu, Barry Design, LLC (United States)

\section{SESSION 10 CHARACTERIZATION SYSTEMS AND APPLICATIONS}

$90801 \mathrm{E}$ The integrated atmospheric characterization system (IACS) [9080-50]

D. W. Roberts, K. R. Albers, E. A. Brown, T. A. Craney, M. M. Hosain, R. K. James, N. D. Meraz, A. J. Mercer, K. D. Nielson, R. L. Ortman, T. W. Pool, J. W. Wood, J. M. Stewart, T. M. Strike, G. G. Gimmestad, Georgia Tech Research Institute (United States); D. N. Whiteman, NASA Goddard Space Flight Ctr. (United States) 
$9080 \mathrm{IF}$ Control of a small robot using a hybrid optical modulating retro-reflector/RF link [9080-52]

J. L. Murphy, M. S. Ferraro, W. S. Rabinovich, P. G. Goetz, M. R. Suite, U.S. Naval Research Lab. (United States); S. H. Uecke, NovaSol (United States)

\section{POSTER SESSION}

$90801 G$ Detecting binary non-return-to-zero data in free-space optical communication systems using FPGAs [9080-53]

V. Bui, L. Tran, E. El-Araby, N. Namazi, The Catholic Univ. of America (United States)

Author Index 


\title{
Conference Committee
}

\author{
Symposium Chair \\ David A. Whelan, Boeing Defense, Space, and Security \\ (United States) \\ Symposium Co-chair
}

Nils R. Sandell Jr., Strategic Technology Office, DARPA (United States)

\section{Part A Laser Radar Technology and Applications XIX}

\section{Conference Chairs}

Monte D. Turner, Air Force Research Laboratory (United States)

Gary W. Kamerman, FastMetrix, Inc. (United States)

Conference Program Committee

Philip Gatt, Lockheed Martin Coherent Technologies (United States)

Dominique Hamoir, ONERA (France)

Richard M. Heinrichs, Defense Advanced Research Projects Agency

(United States)

Robert T. Hintz, Naval Air Warfare Center Weapons Division

(United States)

Norman A. Lopez, FastMetrix, Inc. (United States)

Vasyl Molebny, National Taras Shevchenko University of Kyiv (Ukraine) C. Russell Philbrick, North Carolina State University (United States) Upendra N. Singh, NASA Langley Research Center (United States) Ove K. Steinvall, Swedish Defence Research Agency (Sweden) Douglas G. Youmans, SPARTA Inc./Parsons Corporation

(United States)

\section{Session Chairs}

Advanced Systems and Components I

Monte D. Turner, Air Force Research Laboratory (United States)

Advanced Systems and Components II

Gary W. Kamerman, FastMetrix, Inc. (United States) 
Signal Processing

Vasyl Molebny, National Taras Shevchenko University of Kyiv (Ukraine)

Data Processing and Analysis I

C. Russell Philbrick, North Carolina State University (United States)

Data Processing and Analysis II

Ove K. Steinvall, Swedish Defence Research Agency (Sweden)

Atmospheric Sensing

Douglas G. Youmans, SPARTA Inc./Parsons Corporation

(United States)

\section{Part B Atmospheric Propagation XI}

\section{Conference Chairs}

Linda M. Wasiczko Thomas, U.S. Naval Research Laboratory (United States)

Earl J. Spillar, Air Force Research Laboratory (United States)

Conference Program Committee

Ammar Al-Habash, Raytheon Space \& Airborne Systems (United States)

Gary Baker, Lockheed Martin Space Systems Company (United States)

Harris R. Burris Jr., U.S. Naval Research Laboratory (United States)

Gary G. Gimmestad, Georgia Tech Research Institute (United States)

Ken J. Grant, Defence Science and Technology Organisation (Australia)

Juan C. Juarez, Johns Hopkins University Applied Physics Laboratory (United States)

Christopher I. Moore, U.S. Naval Research Laboratory (United States)

William S. Rabinovich, U.S. Naval Research Laboratory (United States)

Jonathan M. Saint Clair, The Boeing Company (United States)

Douglas G. Youmans, SPARTA Inc./Parsons Corporation

(United States)

David H. Tofsted, U.S. Army Research Laboratory (United States)

Morio Toyoshima, National Institute of Information and Communications Technology (Japan)

Cynthia Y. Young, University of Central Florida (United States) 
Session Chairs

Propagation Theory and Validation

Ammar Al-Habash, Raytheon Space \& Airborne Systems

(United States)

Mitigation Techniques I

Linda M. Wasiczko Thomas, U.S. Naval Research Laboratory

(United States)

Mitigation Techniques II

Harris R. Burris Jr., U.S. Naval Research Laboratory (United States)

Christopher I. Moore, U.S. Naval Research Laboratory (United States)

Characterization Systems and Applications

Juan C. Juarez, Johns Hopkins University Applied Physics Laboratory (United States) 
Proc. of SPIE Vol. $9080908001-12$

Downloaded From: https://www.spiedigitallibrary.org/conference-proceedings-of-spie on 26 Apr 2023 Terms of Use: https://www.spiedigitallibrary.org/terms-of-use 